\title{
A self-management education programme for adults with asthma
}

\author{
Authors: Hamad Dailah, Alison Brettle and Paula Ormandy
}

\section{Introduction}

Globally, asthma is one of the major non-communicable diseases, with 235 million people currently suffering from the condition. In Saudi Arabia, there is a $4 \%$ prevalence of asthma among the adult population. This study aimed to develop an adult asthma patient self-management education programme that took into account the Saudi context and culture.

\section{Systematic review}

A systematic review was conducted to determine the most effective way of delivering asthma self-management education programmes. It was shown in the review that asthma was more prevalent in individuals with less education, and that nurses had an important role in educating patients with this disease. Following analysis of this evidence alongside the Saudi context, small-group education programmes delivered by nurses were selected as the most appropriate means of delivering education, which considered patient individual needs and cultural values to improve their health outcomes in terms of self-management.

\section{Methods}

An explanatory sequential complementary mixed-methods design was adopted. This design encompassed a quasi-experiment using a pre- and post-test comparative study, followed by a qualitative part using face-to-face interviews with patients. The intervention was an educational programme that included topics selected based on patients' needs in the review and following the Saudi Initiative for Asthma (SINA) guidance. 'The programme was tested to determine whether it provide patients with the necessary knowledge and skills to self-manage their asthma and consequently reduce their visits to emergency departments. Further, the programme was approved and co-designed with nursing staff who provide care for patients with asthma in the selected hospital and following consultation with patients. The programme was delivered by nurses and other professionals who were trained to ensure the quality of delivery. Data were collected pre-, post- and at 3 and 6 months following the intervention and included topics related to asthma, Asthma Control Test,
Asthma Self-Management Questionnaire, the Asthma Knowledge Questionnaire and the Patient Activation Measure (PAM) instrument. Prior to data collection, instruments were all translated to Arabic, the language of participants, and validated to assure the validity of patients' responses. Patients in the control group were able to receive the intervention after 6 months, the last data collection point in the study.

\section{Findings}

Analysis of the responses between the pre- and post-tests demonstrate that patients with higher levels of knowledge had higher PAM scores. Patients' scores in all datasets in phase 2 were higher than those in phase 1 , which indicated that the education was effective and increased confidence in patients' self-management of their asthma. Results to date suggest that the bespoke education is relevant and provides the necessary knowledge to increase confidence and patient activation towards disease self-management and reducing complications.

\section{Study progress}

Longer-term follow-up data and qualitative interviews will be completed in late February 2019. It is anticipated that these results will confirm that the educational programme is suited to the Saudi Arabian context, improves knowledge and increases confidence in the self-management of asthma.

\section{Reference}

1 Saudi Initiative for Asthma. Guidelines for the diagnosis and management of asthma in adults and children. Riyadh: Saudi Initiative for Asthma (SINA) Group, 2016. http://sinagroup.org/download/ SINA_Guidelines_2016.pdf [Accessed on 4 April 2019]. 